\title{
Interaction of electromagnetic waves in an ice layer
}

\author{
Vladimir Korochentsev ${ }^{1, *}$, Wei Xue ${ }^{2}$, Gennadiy Shabanov ${ }^{3}$, Artem Em ${ }^{1}$, and Yuliya Shpak ${ }^{1}$ \\ ${ }^{1}$ Far Eastern Federal University, School of Engineering, Department of Construction Engineering \\ Systems,690922, Vladivostok, Russkiy island, Russia \\ ${ }^{2}$ Harbin Engineering University, College of Underwater Acoustical Engineering, 150001, Harbin, \\ Chengen, China \\ ${ }^{3}$ Scientific Research Center «Arktika» FEB RAS, Vladivostok, Kirova st., 95, Russia
}

\begin{abstract}
A mathematical model for spherical wave propagation near an ice layer has been developed. The proposed mathematical model is based on the directed Green's functions with boundary conditions with irregular angles. Based on the suggested model, we analyzed a field of a point directed source radiating electromagnetic waves in two cases: the source is in the air and is radiating waves along the marine ice surface; the source is in the ice layer and radiating waves. Results of the modeling for different frequencies and different ice thickness are described. It was shown that wave amplitude increases by about two times inside the ice if compared to free space. Electromagnetic wave propagating along marine ice surface increases by $50 \%$ compared to the wave propagating in free space.
\end{abstract}

\section{Theory}

Preparation and development of earthquakes is accompanied by magnetic, electric, elastic fields in an earthquake source during accelerated mechanic motion of an earthquake source in the Earth magnetic field with possible generation of electromagnetic waves [2].

Transfer of the data on earthquake source may also be realized by electromagnetic waves propagating in the atmosphere near water and ice surfaces. In the Arctic, owing to high noise, the communication between several correspondents is unstable and unreliable. One of the ways to improve the reliability of communication on electromagnetic wave may be the choice of ice layer as a propagation path. Distribution of electromagnetic waves near an ice cover and inside an ice layer may be carried out by different techniques. The present paper suggests a mathematical algorithm based on the theory of directed Green's functions which allows one to analyze electromagnetic wave field with the accuracy sufficient for practice.

\subsection{Mathematical model}

* Corresponding author: vkoroch@mail.ru 
We choose the Helmholtz equation, describing magnetic and electric components of electromagnetic waves, as a mathematical model for electromagnetic wave propagation.

To simplify the problem, we represent the electromagnetic wave vector components in the form of a potential function. Field potential is expressed by Green's function of free space in a spherical system. Green's function for free space (non- directed Green's function) is written as

$$
\bar{G}_{1}\left(M, M_{0}\right)=\bar{\Phi}_{0} \frac{e^{i k R}}{R}
$$

$R$ is the distance between the points $M$ and $M_{0}$;

Radiation is applied to an ice layer which covers a sea surface (Fig. 1). The following characteristics are known: magnetic and electric permeability of air, sea and ice.

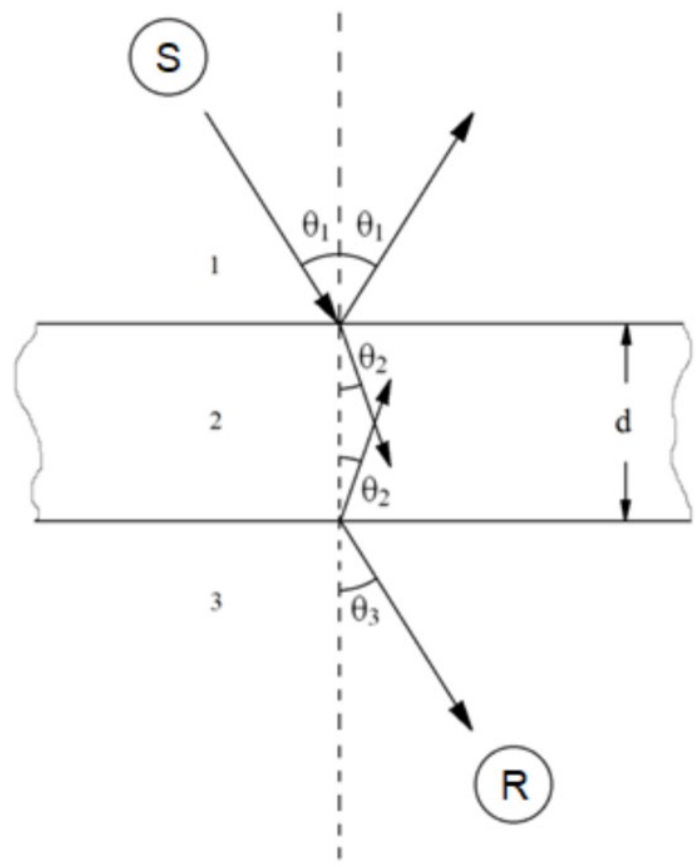

Fig. 1. Geometry of the problem under consideration. $\mathrm{S}$ - radiation source, 1 - air half-space, 2 - ice layer, 3 - water half-space, $\mathrm{R}$ - receiver

It is known that Helmholtz equation equivalent solutions are two conjugated Green's functions. One of Green's functions describes waves $G_{1}\left(M, M_{0}\right)$ diverging from the radiator surface, and the second one $G_{1}^{-1}\left(M, M_{0}\right)$ is conjugated to it [1]. Strictly speaking, the general solution of Helmholtz equation should take into account the sum of these functions

$$
\bar{G}_{\Sigma}=\sum_{l=1}^{L}\left[\bar{G}_{1}\left(M, M_{0}\right)+k_{\mathrm{omp} l} \bar{G}_{1}^{-1}\left(M, M_{0}\right)\right]
$$

$M$ is the point of signal reception with the coordinates $x$ and $y ; M_{0}$ is the point of signal radiation with the coordinates $x_{0}$ and $y_{0}$.

Directed Green's function of free space has the form 


$$
\bar{G}_{l}=\frac{i \bar{\Phi}_{0}}{2 \pi} \int_{U_{\min }}^{U_{i \max }} \frac{F_{l}(\theta)}{\sqrt{k^{2}-U_{l}^{2}}} e^{\left[i\left(x-x_{0}\right) \sqrt{k^{2}-U_{l}^{2}}+\left(y-y_{0}\right) U_{l}\right]} d U_{l} .
$$

where $F_{l}(\theta)=1$, if $\theta_{l} \min \leq \theta \leq \theta_{l} \max$ when $l=1,2,3, \ldots, l . F_{l}=0$ for other $\theta$ values, $U_{l}=k \sin \theta$.

In this paper, spherical waves are presented in the form of superposition of plane waves, thus it is fair to apply the reflection coefficient for plane waves

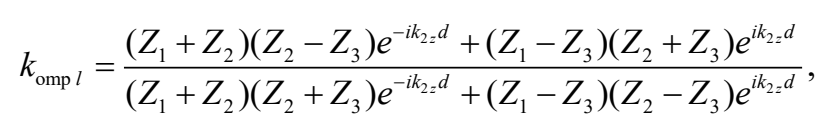

$d$ is the ice layer thickness; $k_{2 z}$ is the wave vector component; $Z_{l}$ is wave resistance of the 1 th medium equal to

$$
Z_{l}=\frac{\varepsilon_{l}}{\mu_{l}} / \cos \theta_{l}
$$

Coefficient of a wave passage from medium 1 to medium 3 is determined as

$$
W=1-k_{\mathrm{omp} l} \text {. }
$$

According to the formulated problem, Green's function for a refraction wave is written as

$$
\bar{G}_{l}=\frac{i \bar{\Phi}_{0}}{2 \pi} W \int_{U_{\min }}^{U_{\text {max }}} \frac{F_{l}(\theta)}{\sqrt{k^{2}-U_{l}^{2}}} e^{\left[i\left(x-x_{0}\right) \sqrt{k^{2}-U_{l}^{2}}+\left(y-y_{0}\right) U_{l}\right]} d U_{l} .
$$

Expression (7) describes a point source field in medium 3.

\subsection{Source in an ice layer}

As it is illustrated in Fig. 2, a point radiation source is located in a closed volume. The volume is compound of three mediums with different parameters of electric and magnetic permeability. The source is located in an ice layer. The wave emitted in medium 2 is rereflected from mediums 1 and 3 many times during the propagation process. Arrows from the source show the emission angle limits.

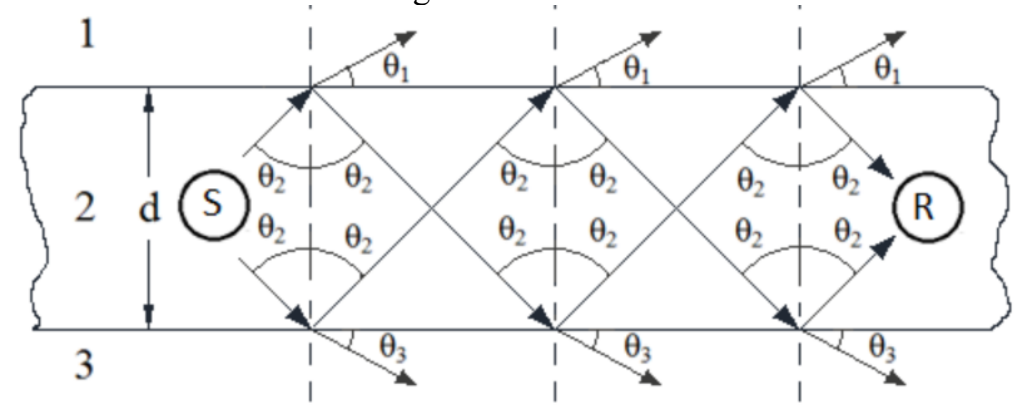

Fig. 2. Geometry of the problem under consideration. $\mathrm{S}$ - radiation source, 1 - air half-space, 2 - ice layer, 3 - water half-space, $\mathrm{R}$ - receiver. 
In this case, the reflection coefficient is written as

$$
k_{\text {omp }}=k_{21} k_{23}
$$

where $k_{21}$ is the coefficient of reflection from medium 1 surface; $k_{23}$ is the coefficient of reflection from medium 3 .

$$
\begin{gathered}
k_{21}=\frac{Z_{1}-Z_{2}}{Z_{1}+Z_{2}}, \\
k_{23}=\frac{Z_{3}-Z_{2}}{Z_{3}+Z_{2}}, \\
k_{\text {omp }}=\frac{\left(Z_{1}-Z_{2}\right)\left(Z_{2}-Z_{3}\right)}{\left(Z_{1}+Z_{2}\right)\left(Z_{2}+Z_{3}\right)}, \\
\bar{G}_{l}=\bar{\Phi}_{0}\left[\int_{U_{l \min }}^{U_{\max }} \frac{F_{l}(\theta)}{\sqrt{k^{2}-U_{l}^{2}}} e^{\left[i\left(x-x_{0}\right) \sqrt{k^{2}-U_{l}^{2}}+\left(y-y_{0}\right) U_{l}\right]} d U_{l}+\right. \\
\left.+k_{\mathrm{omp}}^{U_{l \max }} \frac{F_{l}(\theta)}{\sqrt{k_{l \min }^{2}-U_{l}^{2}}} e^{\left[i\left(x-x_{0}\right) \sqrt{k^{2}-U_{l}^{2}}+\left(y-y_{0}\right) U_{l}\right]} d U_{l}\right] .
\end{gathered}
$$

\subsection{Geometry of the problem of electromagnetic wave propagation in an ice layer}

A point source of electromagnetic waves is located in an ice space at point 0 . The volume is limited by three plane boundaries denoted as $U_{0}, U_{1}, U_{2} . U_{0}$ is the ice-ice interface; $U_{1}$ is the ice-atmosphere interface; $U_{2}$ is the ice-water interface. To calculate source field, we apply directed Green's functions with boundary conditions with irregular angles, $P P O(x), P P S(x)$. Function $P P O(x)$ describes waves in a volume limited by the angle $\theta_{0}$ and the boundary $U_{0}$. Function $P P S(x)$ describes the waves incident to the boundary $U_{1}$ and reflected from $U_{1}$ wave in a volume limited by the angle $\theta_{1}$ and the boundary $U_{1}$, as well as those incident to the boundary $U_{2}$ and reflected from $U_{2}$ wave in the volume limited by the angle $\theta_{2}$ and the boundary $U_{2}$.

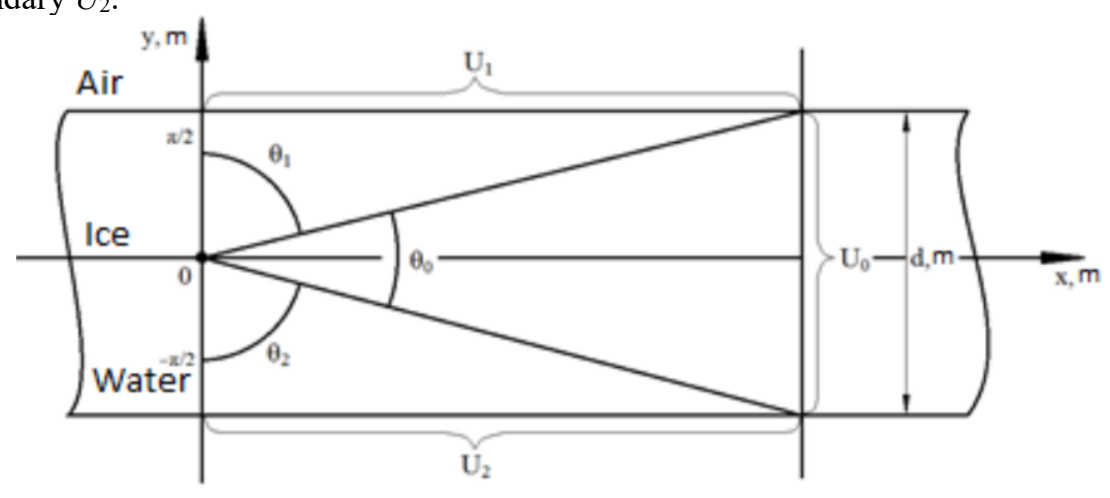

Fig. 3. Ice layer geometry. 


\section{Results of numerical calculations}

Fig. 4-12 show the results of calculations based on the proposed theory.

In a free space, Green's function at the frequency of $f=1 \mathrm{MHz}$ behaves as follows:

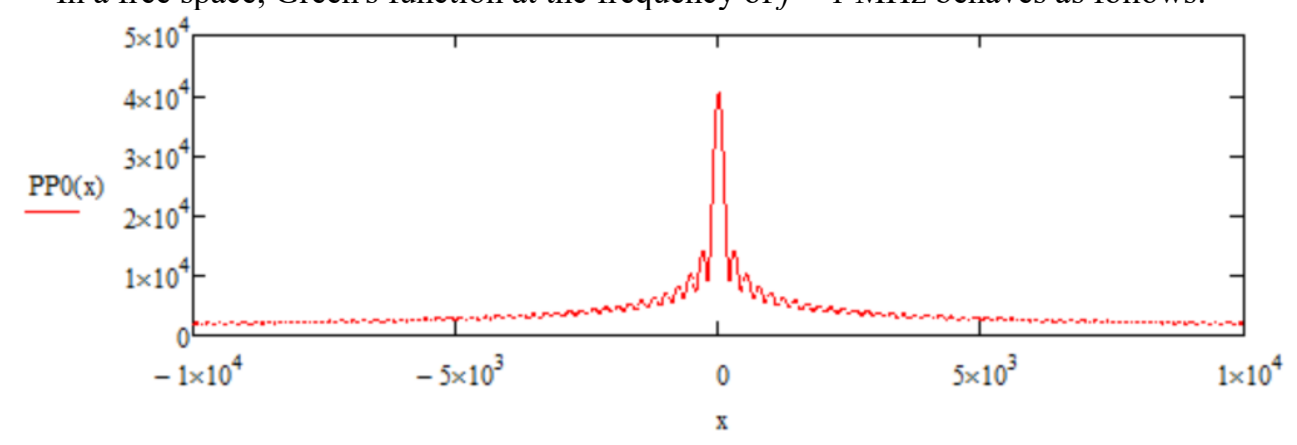

Fig. 4. Graph of electric field strength distribution in an ice space. Initial data of medium characteristics: radiator frequency $f=1 \mathrm{MHz}$; ice layer thickness $d=3 \mathrm{~m}$, distance $10000 \mathrm{~m}$, power $P=4000 \mathrm{~W}$.

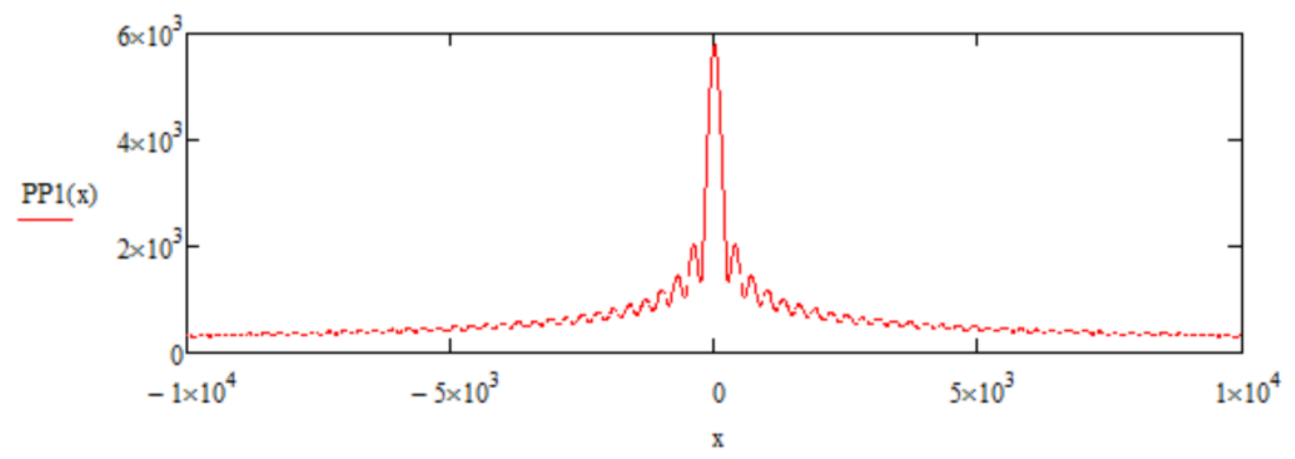

Fig. 5. Graph of electric field strength distribution at ice-atmosphere interface. Initial data of medium characteristics: radiator frequency $f=1 \mathrm{MHz}$; ice layer thickness $d=3 \mathrm{~m}$, distance $10000 \mathrm{~m}$, power $P=4000 \mathrm{~W}$.

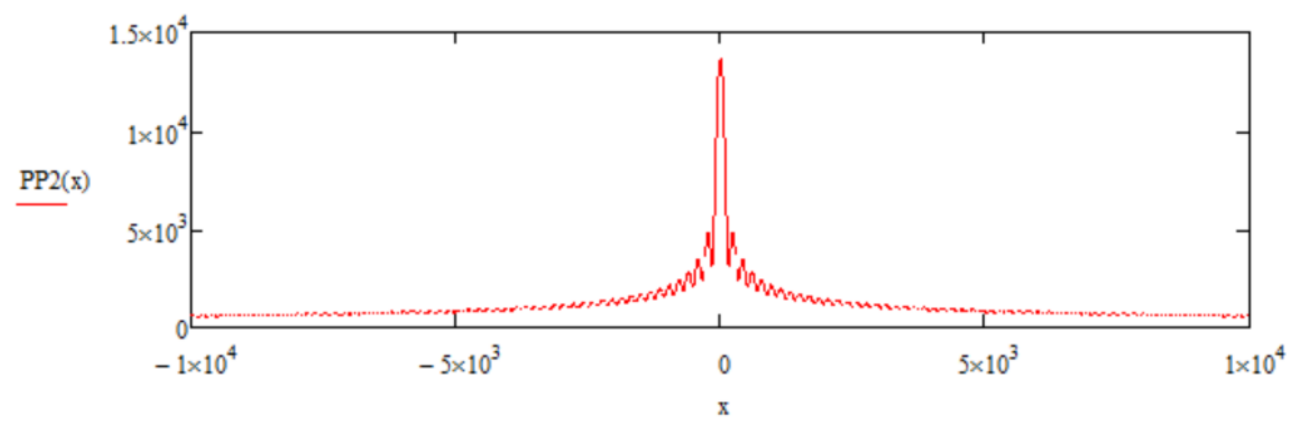

Fig. 6. Graph of electric field strength distribution at ice-atmosphere interface. Initial data of medium characteristics: radiator frequency $f=1 \mathrm{MHz}$; ice layer thickness $d=3 \mathrm{~m}$, distance $10000 \mathrm{~m}$, power $P=4000 \mathrm{~W}$. 


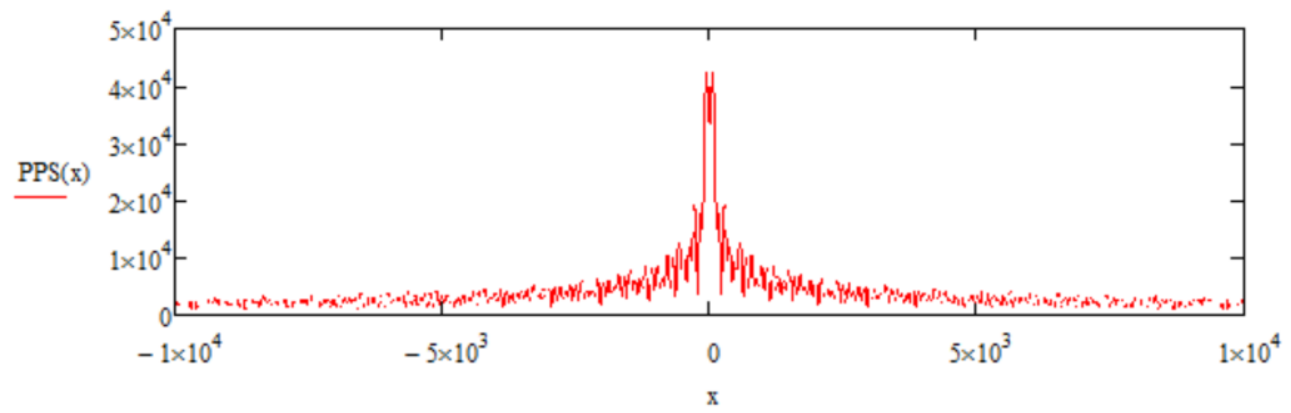

Fig. 7. Sum of Green's functions (field in an ice layer). Initial data of medium characteristics: radiator frequency $f=1 \mathrm{MHz}$; ice layer thickness $d=3 \mathrm{~m}$, distance $10000 \mathrm{~m}$, power $P=4000 \mathrm{~W}$.

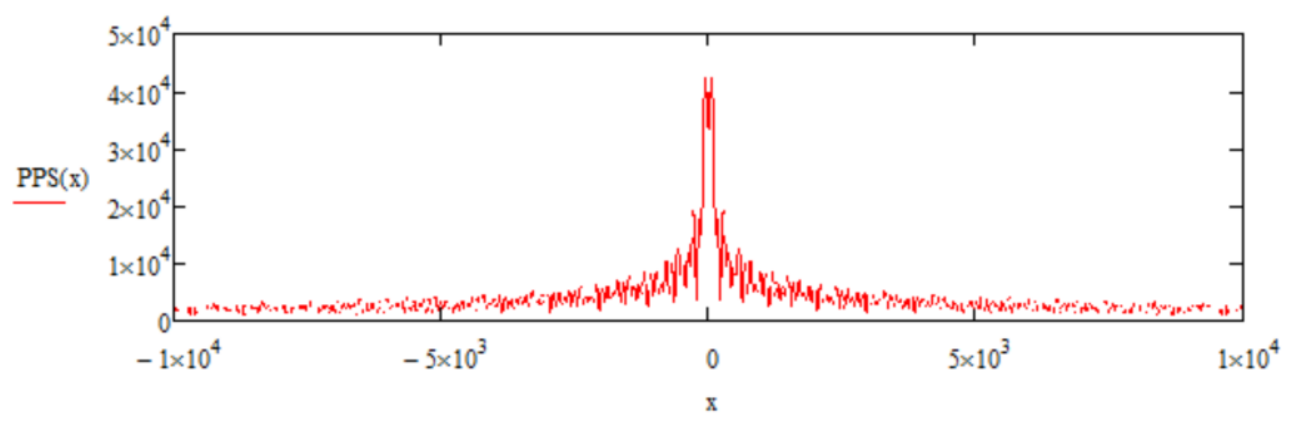

Fig. 8. Sum of Green's functions (field in an ice layer). Initial data of medium characteristics: radiator frequency $f=1 \mathrm{MHz}$; ice layer thickness $d=6 \mathrm{~m}$, distance $10000 \mathrm{~m}$, power $P=4000 \mathrm{~W}$.

In free space, Green's function at the frequency $f=5 \mathrm{MHz}$ behaves as follows:

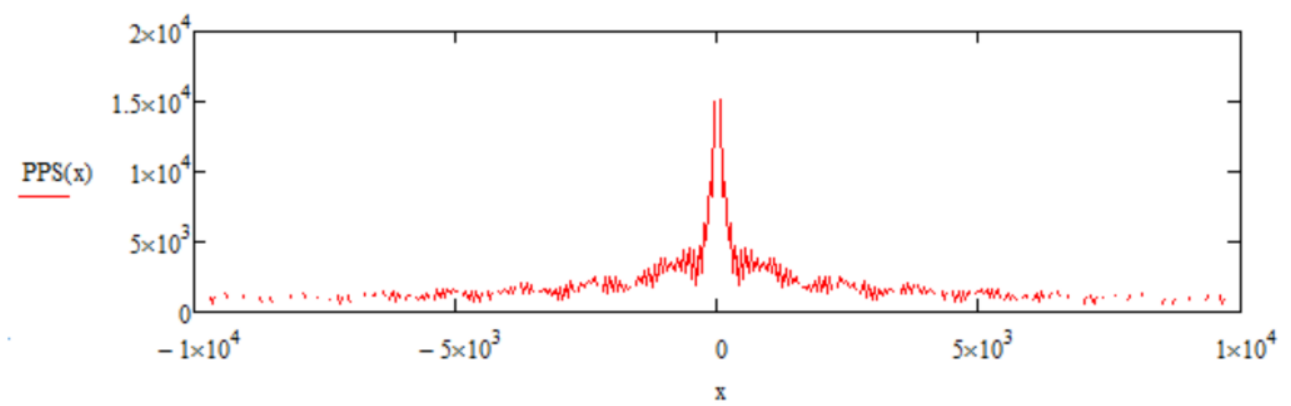

Fig. 9. Sum of Green's functions (field in an ice layer). Initial data of medium characteristics: radiator frequency $f=5 \mathrm{MHz}$; ice layer thickness $d=3 \mathrm{~m}$, distance $10000 \mathrm{~m}$, power $P=4000 \mathrm{~W}$. 


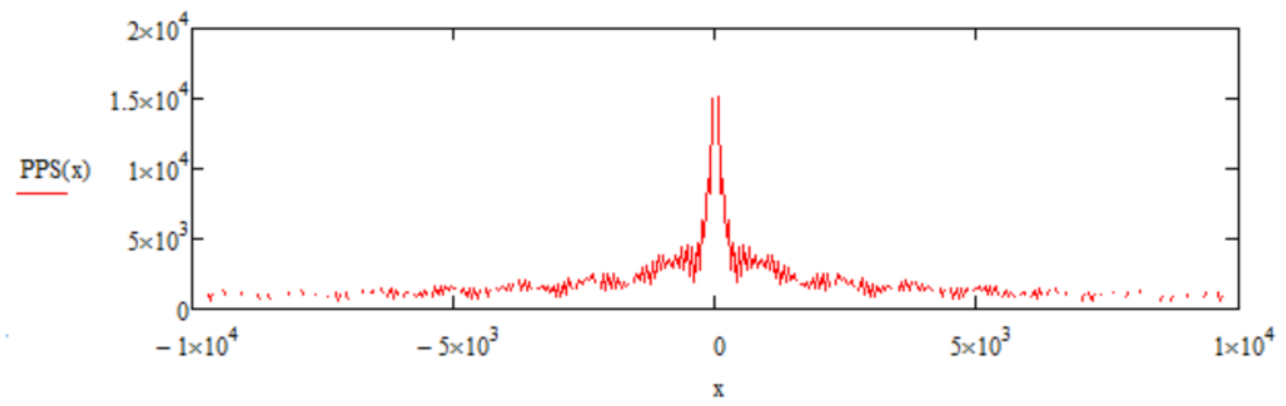

Fig. 10. Sum of Green's functions (field in an ice layer). Initial data of medium characteristics: radiator frequency $f=5 \mathrm{MHz}$; ice layer thickness $d=6 \mathrm{~m}$, distance $10000 \mathrm{~m}$, power $P=4000 \mathrm{~W}$.

In free space, Green's function at the frequency $f=10 \mathrm{MHz}$ behaves as follows:

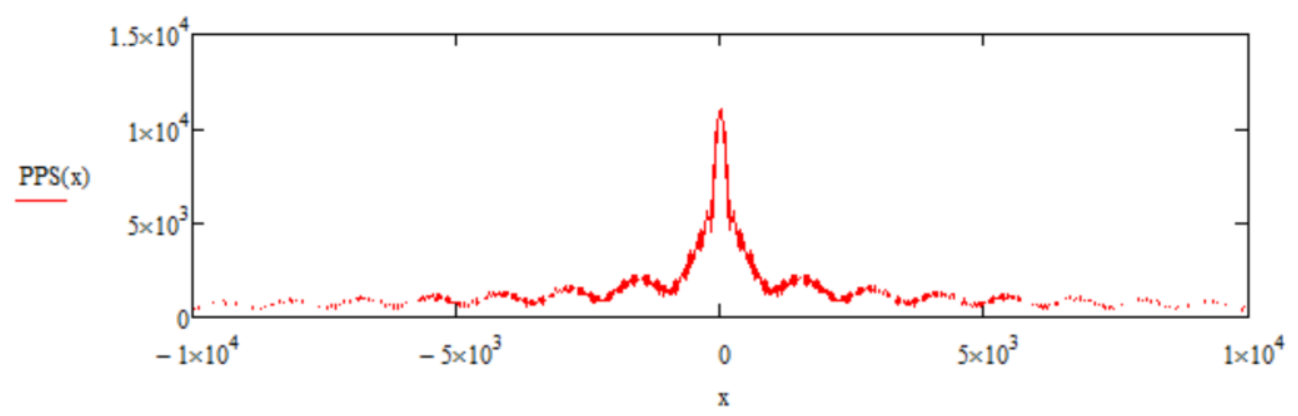

Fig. 11. Sum of Green's functions (field in an ice layer). Initial data of medium characteristics: radiator frequency $f=10 \mathrm{MHz}$; ice layer thickness $d=3 \mathrm{~m}$, distance $10000 \mathrm{~m}$, power $P=4000 \mathrm{~W}$.

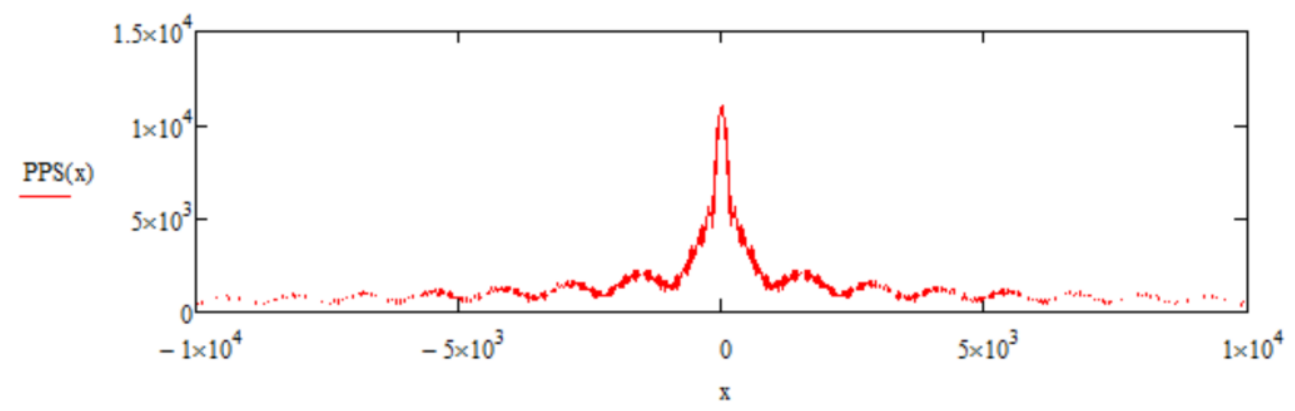

Fig. 12. Sum of Green's functions (field in an ice layer). Initial data of medium characteristics: radiator frequency $f=10 \mathrm{MHz}$; ice layer thickness $d=6 \mathrm{~m}$, distance $10000 \mathrm{~m}$, power $P=4000 \mathrm{~W}$.

To estimate the algorithm error, we make a test calculation of directed Green's function (Fig. 13). Deviations at the boundaries near the angles of $0^{\circ}$ and $90^{\circ}$ are determined by the developed algorithm errors and do not exceed $5 \%$ within $5^{\circ}<\theta<85^{\circ}$. 


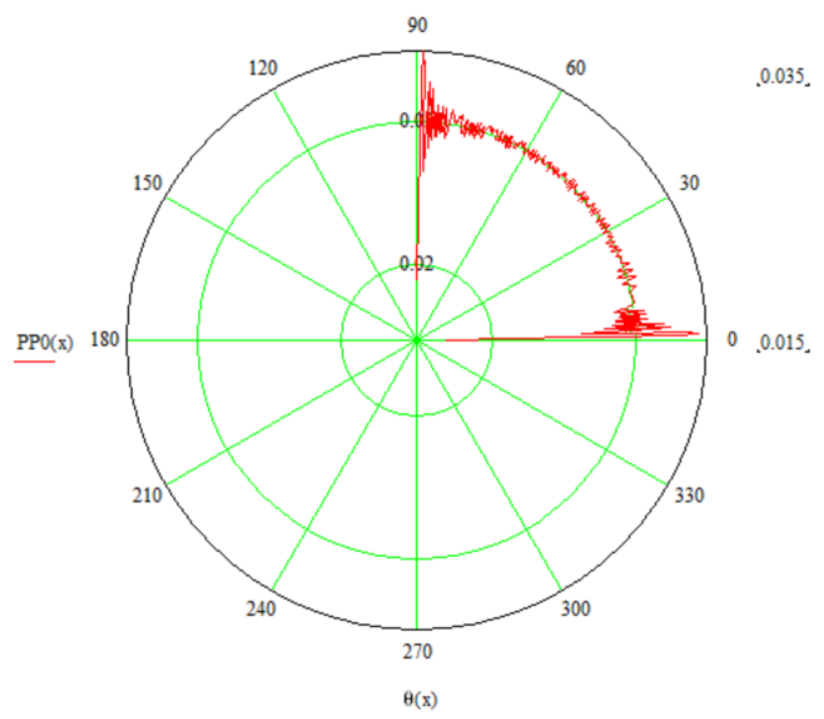

Fig. 13. Directivity pattern of a spherical radiator by formulas (1) and (3).

The main advantage of the method is the calculation rate (203 minutes when calculating Green's function values of 1000 points on ASUS X550 laptop). The proposed algorithm allows us to calculate fields of point electromagnetic radiators in layered mediums taking into account wave reflection from interfaces. The calculation error does not exceed $20 \div 25 \%$.

\section{Conclusions}

In the investigations of surface wave propagation which are used for

- long-distance over-the-horizon radio location;

- detection of low-altitude targets at the heights of less than $100 \mathrm{~m}$;

- $\quad$ ship navigation in confined space, detection of ice field edges, monitoring of ocean surfaces etc.

In Arctic conditions

- investigation of physical properties of Arctic ice;

- electromagnetic wave propagation distance in ice conditions.

Applying wave propagation in ice layer as a trace we can extend the distance of communication systems by about 1.5-2 times as long as electromagnetic field $P(x)$ decreases according to the cylindrical law (Fig. 4-12).

Based on the proposed theory, we can make the conclusion that application of an ice layer as a wave propagation path significantly increases the range of system coverage compared to free space.

\section{References}

1. V.I. Korochentsev, Wave problems of the theory of directed and focusing antennas: monography (Dal'nauka, Vladivostok, 1998)

2. V.V. Korochentsev, M.I. Zvonarev, A technique for forecast of earthquake location and intensity (RF Patent 2147755) 
3. V.V. Korochentsev, M.I. Zvonarev, A technique for forecast of earthquake location and intensity (RF Patent2150717)

4. Yu.V. Marapulets, B. Shevtsov, Mesoscale acoustic emission (Dal'nauka, Vladivostok, 2012)

5. L.M. Brekhovskikh, Waves in layered mediums (Nauka, Moscow, 1973)

6. S.A. Shevkun, Development of methods for analsysis of wave fields in closed volumes: Dissertation of a Cand. Sci. (Vladivostok, 2006)

7. E.L. Shenderov, Wave problems in Hydroacoustics (Sudostroenie, Leningrad, 1972)

8. T.M. Lobova, FEFU: School of Engineering Bulletin, 35 (2018)

9. A.V. Korchaka, FEFU: School of Engineering Bulletin, 38 (2019)

This research was funded by the National Key R\&D Program of China (2018YFC1405900) 